\title{
Using NetMagic to Observe Fine-Grained Per-Flow Latency Measurements
}

\author{
Tao Li \\ School of Computer \\ National University of Defense \\ Technology \\ Changsha, Hunan, China \\ taoli.nudt@gmail.com
}

\author{
Zhigang Sun \\ School of Computer \\ National University of Defense \\ Technology \\ Changsha, Hunan, China \\ sunzhigang@263.net
}

\author{
Chunbo Jia \\ School of Computer \\ National University of Defense \\ Technology \\ Changsha, Hunan, China \\ jiachunbo1988@sina.com
}

\author{
Qi Su \\ School of Computer \\ National University of Defense \\ Technology \\ Changsha, Hunan, China \\ suqi1115@163.com
}

\author{
Myungjin Lee \\ School of Electrical and \\ Computer Engineering \\ Purdue University \\ West Lafayette, USA \\ mjlee@purdue.edu
}

\begin{abstract}
We introduce NetMagic [1] to demonstrate the efficacy of RLI architecture [2] for the fine-grained per-flow latency measurements. In this demo, the main function of RLI is implemented in NetMagic, which is the key component of our experimental network comprising several computers and switches. We are going to show how NetMagic can provide rapid implementation and evaluation of RLI architecture that is difficult with commercial switch or router platforms. In the demo, the estimated fine-grained per-flow latency by RLI is monitored and dynamically presented. Further, the true latency with a resolution of $8 \mathrm{~ns}$ is also provided by NetMagic for the evaluation. The efficacy of RLI architecture can be observed in a real-time fashion by the difference between estimated latencies and true ones.
\end{abstract}

\section{Categories and Subject Descriptors}

C.2.1 [Computer Communication Networks]: Network Architecture and Design; C.2.3 [Computer Communication Networks]: Network Operations-Network monitoring

\section{General Terms}

Design, Experimentation, Measurement

\section{Keywords}

NetMagic Platform, Per-Flow Latency, Measurement, RLI Architecture

\section{INTRODUCTION}

Reference Latency Interpolation (RLI) architecture [2] is proposed for accurately detecting per-flow latencies in the order of a few 10 s to 100 s microseconds. It can be implemented in a router by integrating the adaptive refer-

Copyright is held by the author/owner(s).

SIGCOMM'11, August 15-19, 2011, Toronto, Ontario, Canada.

ACM 978-1-4503-0797-0/11/08. ence packet generation component at a sender side (e.g., an ingress interface) and the measurement component at a receiver (e.g., an egress interface). However, the implementation involves software/hardware modification or upgrade of the router, which may be difficult for router vendors to accept due to reliability and security. Mirroring all the packets from the receiver to a PC, or to a network appliance capable of processing them (such as NetFPGA [3] or high-speed network processor dedicated for these fine-grained measurements) is a way for deploying the RLI. However, the ingress and egress timestamps of the packets are hardly obtained by mirroring way for calculating true latencies of the flows under measurement. In fact, these timestamps are essential for the demo to show the efficacy of the RLI architecture.

To address the above problems, we use an open reconfigurable switching platform - NetMagic [1] to implement a RLI experimental prototype. NetMagic is designed to enable researchers and students to build high-performance networking systems and verify their new ideas and methods for network innovation. Different from the NetFPGA [3], the NetMagic is designed with a novel architecture, where a common high-density FPGA is combined with a commercial Ethernet switching chip. The high-speed Gigabit Ethernet switching capacity, as well as reconfigurable user-defined packet processing function, can be obtained from the NetMagic platform. Secondly, the NetMagic is a standalone network device which is loosely coupled with controller. Standard Ethernet ports and socket interface are utilized for the communication between the remote controller and the NetMagic platform. The NetMagic does not need to be plugged into a specific host machine with related drivers, which simplifies the construction of network experimental systems. Thirdly, the NetMagic provides $2 \mathrm{x}$ reconfigurable ports than the NetFPGA, which is essential for a larger network experimental systems such as this demo.

ServerSwitch [4] is proposed for implementing and evaluating the design of data center networks, which utilizes an ASIC commodity switching chip for data plane and the server CPU for control plane. It thus provides full programmability in the control plane. However, the reconfigurability in data- 
plane is limited, which can not support some specific packet processing functions such as timestamping, digest extraction and arbitrary field modification. For more comparison among the NetMagic, the NetFPGA and the ServerSwitch, see [1].

Like the NetFPGA, to ease the development effort of beginners, the NetMagic platform provides simple and rapid development model, clear module interface and several standard reference designs, which allows users to focus on the implementation of self-defined functional modules. Verified functional components are also provided, including hardware modules, physical interfaces, and software functions. The RLI demo system was developed rapidly by utilizing these reusable functional components of NetMagic.

\section{DEMO OVERVIEW}

Fig. 1 shows the deployment of our demonstration. The demo consists of a NetMagic platform, two Ethernet switches and several PCs or laptops. The device under measurement (DUM) is composed of two 10/100/1000M adaptive Ethernet switches connected by a $100 \mathrm{M}$ link (In fact, only one standalone 10/100/1000M Ethernet switch is used in the demo, because the Ethernet switching chip integrated in the NetMagic functions the same as commercial switches.). PC1, PC2,..., PC5 are computers with 1000M Ethernet interface adaptor. NetMagic connects PC1 and PC2 to the DUM through its $1000 \mathrm{M}$ ports linking to the integrated FPGA. PC3 and PC4 connect with the two Ethernet switches respectively. PC5 connects with NetMagic for receiving, processing and displaying packet latency information.

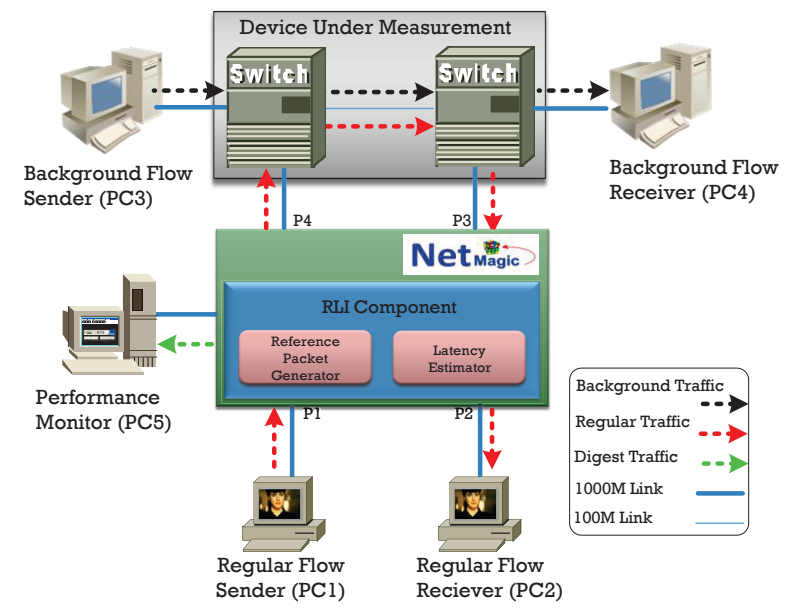

Figure 1: The topology of RLI demonstration.

RLI component is implemented with NetMagic platform for the functions of reference packet generation and latency estimation. The reference packet generator injects reference packets with egress timestamps periodically into the regular packet stream at the egress interface (P4) of NetMagic. It also extracts and sends the digest information (e.g., egress timestamp) of the regular packets to the performance monitor. The ingress timestamps of the reference and regular packets are recorded by NetMagic when the packets return from the DUM (P3). The latency estimator calculates the delay of the reference packets based on their ingress and egress timestamps. It also extracts and sends the digest information with ingress timestamps of the regular packets to the performance monitor. Based on the received information, the performance monitor calculates the estimated delay and the true delay of each regular packet. It then dynamically displays the true delay versus the estimated delay values of every sample packet, as well as the average delay values of the flows. Because all the interfaces of the NetMagic operates within the same time-domain (125MHz Clock), we do not need extra time synchronization for timestamping and the timestamps are with a resolution of $8 \mathrm{~ns}$.

In the demo, we will adopt delay-sensitive streaming media applications to generate regular flows for measurements. $\mathrm{PC} 1$ will send several standard-definition video flows to $\mathrm{PC} 2$. After the packets of the video streams traverse the NetMagic platform and the DUM, PC2 displays them on its screen.

The PC3 and PC4 (with 1000M Ethernet interface) will run Iperf applications [5], where PC3 is set to the UDP client and PC4 is set to UDP server. By changing the parameters of Iperf at the client side, the utilization rate of the $100 \mathrm{M}$ link between the two switches can be adjusted. The 100M link of DUM can be made congested, as PC3 can send UDP packets to PC4 at the speed of up to $1000 \mathrm{Mbps}$.

From PC5, attendees will observe the real-time curves of the true and estimated latency values of the sample packets and the corresponding average values of the buffered packets. They can also watch the videos on the screen of $\mathrm{PC} 2$ for the intuitive experience of video quality. By adjusting the parameters of Iperf application, the attendees can vary the sending rate of background traffic. As a consequence, they will observe the changes of the quality of the video (e.g. smooth, distortion or frequent pauses) from PC2. Correspondingly, the changes of the latency values will be shown on PC5. The accuracy of the RLI architecture is illustrated by the positions and the shapes of the curves indicating the true and estimated latency values. This demo will prove that RLI achieves a high accuracy for the fine-grained per-flow latency measurement if the two dynamic curves fit well.

\section{ACKNOWLEDGMENTS}

The work was supported by China National Basic Research (973) Program (No. 2009CB320503) and China State High-Tech Development (863) Plan (No. 2009aa01a334).

\section{REFERENCES}

[1] NetMagic Research Group. http://www.netmagic.org.

[2] Myungjin Lee, Nick G. Duffield, and Ramana Rao Kompella. Not all microseconds are equal: fine-grained per-flow measurements with reference latency interpolation. In SIGCOMM, pages 27-38, 2010.

[3] John W. Lockwood, Nick McKeown, Greg Watson, and etc. NetFPGA-an open platform for gigabit-rate network switching and routing. In MSE '07, 2007.

[4] Guohan Lu, Chuanxiong Guo, Yulong Li, Zhiqiang Zhou, Tong Yuan, Haitao Wu, Yongqiang Xiong, Rui Gao, and Yongguang Zhang. Serverswitch: a programmable and high performance platform for data center networks. NSDI'11, pages 2-2, Berkeley, CA, USA, 2011.

[5] A. Tirumala, M. Gates, F. Qin, J. Dugan, and J. Ferguson. Iperf - the tcp/udp band- width measurement tool. http://dast.nlanr.net/Projects/Iperf. 